\title{
Anterior chamber lens implantation after vitreous loss*
}

\author{
P ANDREW PEARSON, DOUGLAS G OWEN, MAUREEN MALISZEWSKI, \\ AND THOMAS J SMITH \\ From the Glaucoma Service, University of Kentucky Medical Center, Lexington, Kentucky, USA
}

SUMMARY Vitreous loss is a serious complication of cataract surgery. Following vitreous loss it is common practice to perform a primary implantation of an anterior chamber lens (AC-IOL). We retrospectively analysed 642 consecutive cases of cataract extraction performed between 1983 and 1986 with special attention to those patients in whom vitreous loss occurred and an AC-IOL was placed. There were 27 such cases, and 24 of these were available for follow-up. Eighteen (75\%) had visual acuity of $20 / 40$ or better. All six patients $(25 \%)$ who had a visual acuity of less than $20 / 40$ in the operated eye had a functional visual acuity of 20/200 or less. Complications that occurred in this group are discussed. We are concerned that the complications associated with vitreous loss and with AC-IOLs may be acting in concert to cause visually disabling results.

Vitreous loss is a well known complication of cataract surgery. Following vitreous loss there is an increased risk of retinal detachment, expulsive haemorrhage, cystoid macular oedema, corneal decompensation, and secondary glaucoma. ${ }^{1}$ The visual outcome in these patients is compromised. ${ }^{23}$ After vitreous loss occurs it is common practice to do a partial anterior vitrectomy and then place an anterior chamber lens (AC-IOL). Reports on the effect of placing an ACIOL after vitreous loss have concluded that in experienced hands it is a safe procedure. ${ }^{4-6}$ However, these reports do show that patients who receive an AC-IOL after vitreous loss do significantly less well than those patients who receive a posterior chamber intraocular lens (PC-IOL) after straightforward extracapsular cataract surgery. The contribution of the AC-IOL to this diminished success is unclear at present. One of us (TJS) has been particularly struck by the magnitude of the problems induced by AC-IOLs as seen in a referral glaucoma practice. To estimate the incidence of severe complications following AC-IOL placement after vitreous loss we reviewed all of the cataract extractions performed at the Veterans Administration Hospital in Lexington,

\footnotetext{
*Presented in part at the European Intraocular Lens Implant Society, Jerusalem, 1987.

Correspondence to Thomas J Smith MD, Department of Ophthalmology, University of Kentucky, 800 Rose Street, Lexington, KY 40536-0084, USA.
}

Kentucky, from 1983 through 1986 with special attention to those patients in whom vitreous loss occurred and an AC-IOL was placed.

\section{Results}

Six hundred and forty-two consecutive cataract extractions performed between 1983 and 1986 were reviewed. There were 27 instances of vitreous loss with anterior chamber lens insertion $(4.4 \%) ; 24$ $(89 \%)$ were available for follow-up ranging from three months to more than four years. Of the 24 patients who had an AC-IOL placed after vitreous loss $18(75 \%)$ had a visual acuity of $20 / 40$ or better. However, of the six patients $(25 \%)$ who had a visual acuity of less than $20 / 40$ in the operated eye all had functional visual acuity of $20 / 200$ or less. Five of the six patients had a Kellman multiflex MT4 or MT5 manufactured by Cilco implanted. Patient no. 5 had a Cilco AC2 implanted. Complications that occurred in this group leading to the poor visual outcome are summarised in Table 1.

\section{Case reports}

Case 1. A 70-year-old white male with a history of corneal breakdown from probable herpetic infection in 1945 presented with initial VA of hand motion 596 
Table 1 Breakdown of the complications following vitreous loss and implantation of an anterior chamber intraocular lens

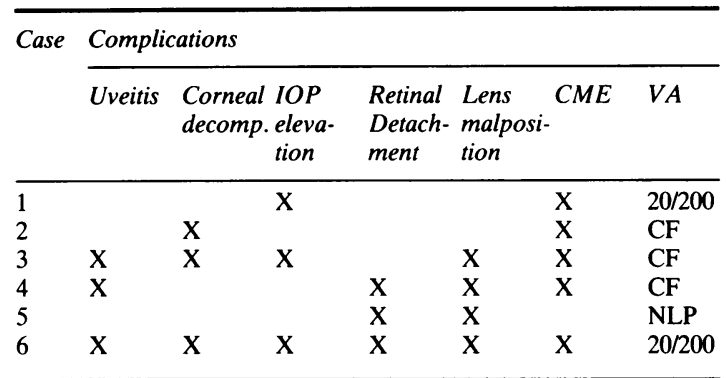

$\mathrm{IOP}=$ intraocular pressure. $\mathrm{CME}=$ chronic cystoid macular oedema. $\mathrm{VA}=$ visual acuity. $\mathrm{CF}=$ counting fingers. $\mathrm{NLP}=$ no light perception.

ulcerative lesion with deep stromal scarring of the cornea OD. A penetrating keratoplasty was performed in combination with an attempted extracapsular cataract extraction (ECCE), which was complicated by vitreous loss. After a partial anterior vitrectomy an AC-IOL was placed. During a followup period of two years he never attained a visual acuity better than 20/200 with correction. He had pupillary block glaucoma which led to chronic angle closure. In addition he suffered from chronic cystoid macular oedema. At the last follow-up, 27 months after surgery, he had a best corrected visual acuity of 20/200.

Case 2. A 77-year-old white male with a history of a corneal ulcer from a chemical burn in 1943 presented two years after a penetrating keratoplasty with an initial visual acuity of 20/200 OS. He underwent an ECCE which was complicated by vitreous loss. After a vitrectomy an AC-IOL was inserted. During the follow-up period of three years his visual acuity was never better than 20/300. His course was complicated by graft failure and cystoid macular oedema. Two repeat penetrating keratoplasty procedures were performed. At the last follow-up he had a visual acuity of counting fingers at $1 \mathrm{~m}, 2-3+$ cystoid macular oedema, and $2+$ corneal oedema, with graft rejection.

Case 3. A 63-year-old white male presented with no significant medical history and a best corrected visual acuity with a pinhole of 20/70 OS. He underwent an ECCE, which was complicated by vitreous loss and had primary implantation of an AC-IOL. During a follow-up period of four years his course was complicated by chronic iritis, corneal oedema, vitritis, and a raised IOP. His best postoperative visual acuity was $20 / 200$. He had 3-4+ inflammatory deposits on the IOL and had a peaked iris with atrophy secondary to haptic traction on the iris. Eleven months after surgery the inferior haptic was in the iris and the superior haptic was floating free. A cellophane maculopathy was present, and the IOL was subsequently removed. At his last follow-up the visual acuity was counting fingers at $60 \mathrm{~cm}$. He had $4+$ cystoid macular oedema, diffuse corneal oedema, and an unstable intraocular pressure.

Case 4: A 68-year-old white male presented with an initial visual acuity of $20 / 400$. He underwent an ECCE complicated by vitreous loss and had an ACIOL placed. His initial postoperative course was complicated by persistent iritis and vitreous haemorrhage. The superior footplates of the lens were noted to be in the iris at three months. At six months he was noted to have cystoid macular oedema and vitreous haemorrhage, and the IOL was subsequently removed. Twenty-five months after the operation he had a retinal detachment, which was successfully repaired. At his last follow-up 35 months after surgery he had a visual acuity of counting fingers at $60 \mathrm{~cm}$.

Case 5. A 71-year-old white male presented with an initial visual acuity of light perception OS. He underwent an ECCE OS complicated by vitreous loss and had an AC-IOL placed. His initial postoperative visual acuity was $20 / 70$ with a pinhole. The eye was subsequently found to be hypotonous and inferior choroidals were present. Visual acuity decreased to counting fingers at $60 \mathrm{~cm}$. Three months after operation he suffered a retinal detachment. Following repair the diagnosis of 'tight lens syndrome' and pupillary block was made, and the lens was removed. His subsequent course was complicated by rubeosis and progression to a total, inoperable retinal detachment. He had a retrobulbar block for pain control two years after surgery. At his last follow-up the visual acuity was no light perception OS.

Case 6. A 67-year-old white male presented with an initial visual acuity of $20 / 100$. He underwent an ECCE complicated by vitreous loss, and an AC-IOL was inserted. Initial postoperative examination showed a $10 \%$ hyphaema. The pupil was slightly irregular, and his intraocular pressure was increased. The visual acuity was $20 / 200$. His subsequent postoperative course was notable for multiple complications including malpositioning of the IOL, retinal detachment requiring surgical repair, chronic iritis, and chronic cystoid macular oedema. At his last follow-up visit he had a visual acuity of 20/200, 3+ guttae, and early bullous keratopathy. One of the IOL haptics was located in a peripheral iridotomy, and the IOL itself was covered with pigment.

\section{Discussion}

Vitreous loss at the time of surgery can lead to visual impairment. Early reports demonstrate the compli- 
cations that can result from vitreous loss. ${ }^{7-9}$ Although modern management has improved on these results, patients on whom vitreous loss occurs still do less well than patients who do not have this complication. ${ }^{23}$ Kasner and $\mathrm{Jaffe}^{10}$ reported the results on 105 patients with vitreous loss treated by a partial anterior vitrectomy. In this group $81 \%$ had a visual acuity of $20 / 50$ or better. Only $10 \cdot 5 \%$ had vision of $20 /$ 100 or worse.

The role that placement of an AC-IOL after vitreous loss has on the incidence of complications and resultant visual outcome has not been clearly established. Jaffe $\mathrm{J}^{5}$ reported on 88 patients who received IOLs after vitreous loss. Visual acuity was better than $20 / 40$ in about $80 \%$ of them. He concluded that IOL placement after vitreous loss was justified if the surgeon was experienced and that the incidence of complications was not high enough to contraindicate lens implantation. All but one of the IOLs used in this retrospective study were the Binkhorst iris clip lens, which has now become obsolete. Mazzocco ${ }^{4}$ reported a series of 38 patients who had IOLs placed after vitreous loss. Twentythree of these had the Choyce mark VIII placed; in these he noted an increased incidence of glaucoma. Nine of the 23 had glaucoma; all were medically controlled. He came to the same conclusion as did Jaffe. Nishi ${ }^{6}$ reported on 18 patients who had IOLs placed after vitreous loss. He used both anterior and posterior chamber lenses. The final visual acuity in this group ranged from $20 / 60$ to $20 / 15$, while in his control group it was 20/50 to 20/15. He also concluded that placement of an IOL after vitreous loss was not contraindicated.

This series consists of 24 consecutive patients in whom an AC-IOL was placed after vitreous loss and for whom at least three months of follow-up is available. Eighteen of the patients $(75 \%)$ achieved a visual acuity of $20 / 40$ or better, which is in fairly close agreement with Jaffe's results ${ }^{5}$ and similar to that found after vitreous loss alone. ${ }^{23}$ The remaining six patients, however, had multiple complications and a poor visual outcome (visual acuity 20/200 or worse). Some of these patients had complications directly attributable to the AC-IOL, which we believe may have contributed to their poor visual results.

Several of the complications known to occur after vitreous loss are also seen with the use of anterior chamber lenses. Glaucoma, uveitis, and corneal disease are all increased after vitreous loss. These complications are also seen with the use of an ACIOL in uncomplicated surgery. ${ }^{11}$ Mazzocco's results ${ }^{4}$ indicate that there may be a combined effect of the two in the case of glaucoma, but no other study indicates that vitreous loss and an AC-IOL combined yield a significantly increased risk of these complica- tions. Three of the patients reported on here (cases 1 , 3 , and 6) suffered from increased intraocular pressure. In case 1 this was caused by a pupillary block mechanism; the iris was noted to be prolapsing round the lens despite the presence of a peripheral iridectomy. Peripheral anterior synechiae were present. Case 3 had open-angle glaucoma thought to be secondary to chronic iritis, possibly from a malpositioned lens. Three patients (cases 3, 4, and 6) had uveitis. In all of these cases the lens was impinging on the iris. Three of the patients had evidence of corneal decompensation (cases 2, 3, and 6). Case 3 had diffuse corneal oedema after removal of the IOL. This may have been secondary to chronic uveitis or the trauma of lens removal.

An increased risk of retinal detachment follows vitreous loss. ${ }^{111-14}$ While there is no evidence that the use of an IOL increases this risk, the presence of an AC-IOL may make reattachment of the retina more difficult. Three patients had a retinal detachment (cases 4,5 , and 6). In case 4 this occurred several months after the removal of the IOL. In patient 5 the detachment was thought to be inoperable, and the visual acuity progressed to no light perception.

The incidence of cystoid macular oedema is increased after vitreous loss. ${ }^{1}$ Whether an IOL alone increases the risk of this complication is not well established. Jaffe's results ${ }^{5}$ seem to indicate an increased risk in those patients with an iris supported lens, but we are not aware of any study of anterior chamber lenses in association with this complication. Five of our patients had evidence of cystoid macular oedema (cases 1, 2, 3, 4, and 6).

We are concerned about the poor visual outcome in those patients who had complications following vitreous loss and placement of an AC-IOL. As in other series, however, the number of patients is very small. We do not think that a general statement concerning the safety of this practice would be valid. More than a million cataract extractions are performed every year in the United States alone ${ }^{15}$; tens of thousands will suffer the complication of vitreous loss. We believe the complications associated with vitreous loss and AC-IOLs may be acting in concert to cause visually disabling results in some of these patients and that a large prospective study is warranted to delineate the effect an IOL has after vitreous loss. If the incidence of complications is proved to be increased, the surgeon and patient will be better able to weigh the benefits of an IOL after vitreous loss against the possible risk.

\footnotetext{
References

1 Jaffe NS. Cataract surgery and its complications. St Louis: Mosby, 1984.
} 
2 Berger BB, Zweig KO, Peyman GA. Vitreous loss managed by anterior vitrectomy. Arch Ophthalmol 1980; 98: 1245-7.

3 Troutman RC, Clahane AC, Emery JM, et al. Cataract survey of the cataract phacoemulsification committee. Ophthalmology 1975; 79: 178-85.

4 Mazzocco TR. Intraocular lens implantation following vitreous loss. Int Ophthalmol Clin 1979; 19: 155-64.

5 Jaffe NS. Surgical results of cataract and lens implant surgery. In: Klein EA, ed. Symposium on cataract surgery, Trans New Orleans Acad Ophthalmol. St Louis: Mosby, 1983: 226-35.

6 Nishi O. Vitreous loss in posterior chamber lens implantation. $J$ Cataract Refract Surg 1987; 13: 424-7.

7 Vail D. After results of vitreous loss. Am J Ophthalmol 1965; 59: 573-86.

8 Vail D. Loss of vitreous during cataract surgery. Highlights Ophthalmol 1968; 11: 107-19.

9 Dunphy EB. Loss of vitreous in cataract extraction. JAMA 1927; 89: 2254-7.
10 Kasner D. In: Jaffe NS, ed. Cataract surgery and its complications. St Louis: Mosby, 1984: 343.

11 Apple DJ, Mamalis N, Loftfield K, et al. Complications of intraocular lenses. A historical and histopathological review. Surv Ophthalmol 1984; 29: 1-54.

12 Hughes WF, Owens WC. Extraction of senile cataract: a statistical comparison of various techniques and the importance of preoperative survey. Am J Ophthalmol 1945; 28: 40-9.

13 Bagley $\mathrm{CH}$. Retinal detachment: survey of the etiology and results of treatment on phakics and aphakics. Am J Ophthalmol 1948; 31: 285-98.

14 Shapland CD. Retinal detachment in aphakia. Trans Ophthalmol Soc UK 1934; 54: 176-96.

15 Coopersmith LW, Carr MA. Forecasting cataract surgeries and intraocular lens implantation to 1990 using a model of population dynamics. J Cataract Refract Surg 1987; 13: 302-8.

Accepted for publication 20 January 1989. 